\title{
Replacing dietary saturated with unsaturated fat reduces the cardiovascular risk score in men and women at moderate cardiovascular risk: DIVAS study
}

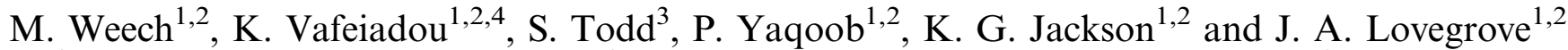 \\ ${ }^{1}$ Hugh Sinclair Unit of Human Nutrition, ${ }^{2}$ Institute for Cardiovascular and Metabolic Research, ${ }^{3}$ Department of \\ Mathematics and Statistics, University of Reading, Reading, RG6 6AP and ${ }^{4}$ School of Life and Medical Sciences, \\ University of Hertfordshire, Hatfield, AL10 9AB
}

A key public health strategy to lower cardiovascular disease (CVD) risk is the reduction of saturated fatty acid (SFA) intake to $\leq 10 \%$ of total energy $(\% \mathrm{TE})^{(1)}$. Replacement of SFA with unsaturated fats may be more beneficial than carbohydrates, ${ }^{(2)}$ yet the optimal class of fatty acid is unclear.

The Dietary Intervention and VAScular function (DIVAS) study developed a food-exchange model to replace $8 \%$ TE dietary SFA with either monounsaturated (MUFA) or n-6 polyunsaturated fatty acids (PUFA) to determine the effects of the intervention on CVD risk score in adults at moderate CVD risk. This 16-wk, parallel, randomised, controlled, single-blinded dietary intervention (ClinicalTrials.gov: NCT01478958), randomly assigned UK adults aged 21-60 years ( $n$ 195) with moderate CVD risk to follow one of three isoenergetic diets providing 36\%TE total fat (\%TE target intakes for SFA:MUFA:n-6 PUFA): SFA-rich (17:11:4; $n$ 65), MUFA-rich $(9: 19: 4 ; n$ 64), or n-6 PUFA-rich $(9: 13: 10 ; n$ 66). A flexible food-exchange model was developed to replace accessible fats in the habitual diet with study foods (spreads, oils, snacks and dairy products) of specific fatty acid composition. Compliance to the dietary regimen was determined by 4-day weighed diet diaries, plasma phospholipid (PL) fatty acid composition and anthropometric measurements at baseline (wk 0) and post-intervention (wk 16). CVD risk score was calculated at screening, wk 0 and wk 16 using a risk assessment tool including raised total cholesterol, glucose and blood pressure, low high-density lipoprotein cholesterol, overweight/obesity and/or family history of premature myocardial infarction or type 2 diabetes. ${ }^{(3)}$ A risk score of $\geq 2$ points was required for participation and reflected a $\geq 50 \%$ greater CVD risk relative to the general population.

Target intakes were largely met for each intervention group. Both dietary and plasma PL fatty acids showed significant overall diet effects for changes in SFA, MUFA and n-6 PUFA between groups after 16 wk $(P<0 \cdot 001)$, except plasma PL n-6 PUFA. Moreover, changes in total fat, protein, carbohydrate, alcohol and BMI did not differ significantly between groups. Relative to baseline, the increase in CVD risk score in the SFA group (0.46 (SEM 0.14) points) was significantly different from the MUFA (0.00 ( $0 \cdot 10)$ points; $P=0.010)$ and n-6 PUFA groups $(-0 \cdot 14(0 \cdot 16)$ points; $P=0.001)$. Weak yet significant correlations between the changes in CVD risk score and dietary SFA $(r=0.216 ; P=0.003)$ and MUFA $(r=-0.143 ; P=0.048)$ were observed, but the negative trend with n-6 PUFA was not significant.

In conclusion, the long-term replacement of SFA with MUFA or n-6 PUFA using a food-exchange model largely achieved target fatty acid intakes, whilst having minimal impact on other nutrients or body weight. The detrimental effect of dietary SFA on CVD risk scores was attenuated or reduced following replacement with MUFA and n-6 PUFA, respectively, in adults at moderate CVD risk. This data supports current public health recommendations to reduce dietary SFA intake as a strategy for CVD risk reduction.

Funded by the Food Standards Agency \& Department of Health Policy Research Programme (024/0036).

1. Department of Health (1994) Nutritional Aspects of Cardiovascular Disease. London: TSO.

2. Kodama S, Saito K, Tanaka S et al. (2009) Diabetes Care 32, 959-965.

3. Chong MF, George TW, Alimbetov D et al. (2013) Eur J Nutr 52, 361-378. 